\title{
On wiring and tiling diagrams related to bases of tropical Plücker functions
}

\author{
Vladimir I. Danilov ${ }^{1}$, Alexander V. Karzanov ${ }^{2}$ and Gleb A. Koshevoy ${ }^{1}$ \\ ${ }^{1}$ Central Institute of Economics and Mathematics of the RAS, 47, Nakhimovskii Prospect, 117418 Moscow, Russia; \\ emails:danilov@cemi.rssi.ru(V.I.Danilov); koshevoy@cemi.rssi.ru (G.A. Koshevoy) \\ ${ }^{2}$ Institute for System Analysis of the RAS, 9, Prospect 60 Let Oktyabrya, 117312 Moscow, Russia; \\ email: sasha@cs.isa.ru.
}

We consider the class of bases $B$ of tropical Plücker functions on the Boolean $n$-cube such that $B$ can be obtained by a series of flips from the basis formed by the intervals of the ordered set of $n$ elements. We show that these bases are representable by special wiring diagrams and by certain arrangements generalizing rhombus tilings on a zonogon.

Keywords: Plücker relations, octahedron recurrence, wiring diagram, rhombus tiling, TP-mutations

\section{Introduction}

This paper deals with bases of tropical Plücker functions defined on a Boolean (hyper)cube and is devoted to a combinatorial description of a wide class of such bases via a relationship to certain classes of wiring and rhombus tiling diagrams.

For a positive integer $n$, let $[n]$ denote the ordered set of elements $1,2, \ldots, n$. Consider a real-valued function $f$ on the subsets of $[n]$, or on the Boolean cube $2^{[n]}$. Following [1], $f$ is said to be a tropical Plücker function, or a TP-function for short, if it satisfies

$$
f(X i k)+f(X j)=\max \{f(X i j)+f(X k), f(X i)+f(X j k)\}
$$

for any triple $i<j<k$ in $[n]$ and any subset $X \subseteq[n]-\{i, j, k\}$. Hereinafter for brevity we write $X i^{\prime} \ldots j^{\prime}$ instead of $X \cup\left\{i^{\prime}\right\} \cup \ldots \cup\left\{j^{\prime}\right\}$. The set of TP-functions on $2^{[n]}$ is denoted by $\mathcal{T P}_{n}$.

Definition. A collection $B \subseteq 2^{[n]}$ is called a TP-basis, or simply a basis, if the restriction map res : $\mathcal{T P}_{n} \rightarrow \mathbb{R}^{B}$ is a bijection. In other words, each TP-function is determined by its values on $B$, and the values on $B$ can be chosen arbitrarily.

Such a basis does exist and the simplest instance is the set $\mathcal{I}_{n}$ of all intervals $\{p, p+1, \ldots, q\}$ in $[n]$ (including the empty set); see, e.g., [2]. In particular, the dimension of the polyhedral conic complex $\mathcal{T P}_{n}$ is equal to $\left|\mathcal{I}_{n}\right|=\left(\begin{array}{c}n+1 \\ 2\end{array}\right)+1$. The basis $\mathcal{I}_{n}$ is called standard.

(Note that the notion of a TP-function is extended to other domains, of which most popular are an integer box $\mathbf{B}^{n, a}:=\left\{x \in \mathbb{Z}^{[n]}: 0 \leq x \leq a\right\}$ for $a \in \mathbb{Z}^{[n]}$ and a hyper-simplex $\Delta_{n}^{m}:=\{S \subseteq[n]:|S|=$ $m\}$ for $m \in \mathbb{Z}$ (in the later case, (1.1) should be replaced by a relation on quadruples $i<j<k<\ell$ ). 
Aspects involving TP-bases or related objects are encountered in [1, 5, 8, 9, 10, 11, 12] and some other works. Generalizing some earlier known examples, [2] constructs a TP-basis for a "truncated integer box" $\left\{x \in \mathbf{B}^{n, a}: m \leq x_{1}+\ldots+x_{n} \leq m^{\prime}\right\}$, where $0 \leq m \leq m^{\prime} \leq a_{1}+\ldots+a_{n}$. The domains different from Boolean cubes are beyond this paper; for some generalizations, see [3].)

Once we are given a basis $B$, we can produce more bases by making a series of elementary transformations relying on 1.1. More precisely, suppose $(X, i, j, k)$ is a cortege such that the four sets occurring in the right hand side of 11.1 and one set $Y \in\{X j, X i k\}$ in the left hand side belong to $B$. Then the replacement in $B$ of $Y$ by the other set $Y^{\prime}$ in the left hand side results in a basis $B^{\prime}$ as well (and we can further transform the latter basis in a similar way). The basis $B^{\prime}$ is said to be obtained from $B$ by the flip (or mutation) with respect to $X, i, j, k$. When $X j$ is replaced by $X i k$, the flip is called raising; otherwise the flip is called lowering. The standard basis $\mathcal{I}_{n}$ does not admit lowering flips, whereas its complementary basis co- $\mathcal{I}_{n}:=\left\{[n]-I: I \in \mathcal{I}_{n}\right\}$ does not admit raising flips.

We further distinguish between two sorts of flips, inspiring consideration of two classes of bases.

Definitions. For a TP-basis $B$ and a cortege $(X, i, j, k)$ as above, the corresponding flip is called strong if both sets $X$ and $X i j k$ belong to $B$ as well, and weak in general. A basis is called normal (in terminology of [2]) if it can be obtained by a series of strong flips starting from $\mathcal{I}_{n}$. A basis is called semi-normal if it can be obtained by a series of weak flips starting from $\mathcal{I}_{n}$.

Leclerc and Zelevinsky [8] showed that the normal bases (in our terminology) are exactly the collections $C \subseteq 2^{[n]}$ of maximum possible size $|C|$ that possess the strong separation property (defined later). Also the normal bases admit a nice "graphical" representation, even for a natural generalization to the integer boxes (see [2, 4]): such bases correspond to the rhombus tilings on the related zonogon.

The purpose of this paper is to characterize the class of semi-normal TP-bases for the Boolean cube $2^{[n]}$, denoted as $\mathcal{B}_{n}$. (It should be noted that it is still open at present whether there exists a non-seminormal, or "wild", basis; we conjecture that there is none.) We give two characterizations for $\mathcal{B}_{n}$ : via a bijection to special collections of curves, that we call proper wirings, and via a bijection to certain graphical arrangements, that we call generalized tilings, or g-tilings for short (in fact, these characterizations are interrelated via planar duality). We associate to a proper wiring $W$ (a g-tiling $T$ ) a certain collection of subsets of $[n]$ called its spectrum. It turns out that proper wirings and g-tilings are rigid objects, in the sense that any of these is determined by its spectrum (see Theorem 3.3 .

Roughly speaking, by a wiring we mean a set of $n$ directed non-self-intersecting curves $w_{1}, \ldots, w_{n}$ in a region $R$ of the plane homeomorphic to a circle, where each $w_{i}$ begins at a point $s_{i}$ and ends at a point $s_{i}^{\prime}$, and the points $s_{1}, \ldots, s_{n}, s_{1}^{\prime}, \ldots, s_{n}^{\prime}$ are different and occur in this order in the boundary of $R$. A special wiring $W$ is defined by three axioms (W1)-(W3). Axiom (W1) is standard, it says that $W$ preserves (topologically) under small deformations. (W2) says that the common points of $w_{i}, w_{j}$ follow in the opposed orders along these wires. The crucial axiom (W3) says that in the planar graph induced by $W$, there is a certain bijection between the faces whose boundary is a directed cycle and the regions ("lenses") surrounded by pieces of two wires between their consecutive common points. $W$ is called proper if none of "cyclic" faces is a whole lens. The spectrum of $W$ is the collection of subsets $X \subseteq[n]$ associated to the "non-cyclic" faces $F$, where $X$ consists of the elements $i$ such that $F$ "lies on the left" from $w_{i}$. When any two wires intersect exactly once, the dual planar graph is realized by a rhombus tiling, and vice versa (for a more general result of this sort, see [6]). The construction of a g-tiling is more intricate. Axiom (W2) occurs in [9]. Another sort of wirings, related to hyper-simplexes, is studied in [9, 10].

Our main result is the following 
Theorem 1.1 For $B \subseteq 2^{[n]}$, the following statements are equivalent:

(i) $B$ is a semi-standard TP-basis;

(ii) $B$ is the spectrum of a proper wiring;

(iii) $B$ is the spectrum of a generalized tiling.

The paper is organized as follows. Section 2 gives precise definitions of proper wirings and generalized tilings. Section 3 outlines ideas of the proof of Theorem 1.1. It consists of four subsections, concerning implications (i) $\rightarrow$ (iii), (iii) $\rightarrow$ (i), (iii) $\rightarrow$ (ii), and (ii) $\rightarrow$ (iii), respectively. (In fact, g-tilings are the central objects of treatment; we take advantages from their nice graphical visualization and structural features, and all implications that we explicitly prove involve just g-tilings. Another advantage of g-tilings is that they admit "local" defining axioms; see the Remark in Section 2, )

Complete proofs of the above-mentioned results are given in the full version [3] of this paper. Moreover, combinatorial methods and technical tools elaborated in those proofs give rise to additional results presented there. Apparently the most important among them is the affirmative answer to a conjecture of Leclerc and Zelevinsky [8] on weakly separated set-systems having maximum possible cardinality.

A commentary: For $A, B \subseteq[n]$, let us write $A \prec B$ if $i<j$ for any $i \in A$ and $j \in B$. Following [8], a pair $A, B$ is called strongly separated if $A-B \prec B-A$ or $B-A \prec B-A$, and is called weakly separated if, up to renaming $(A, B)$ as $(B, A)$, one holds: $|A| \geq|B|$ and $B-A$ can be partitioned into a disjoint union $B^{\prime} \sqcup B^{\prime \prime}$ so that $B^{\prime} \prec A-B \prec B^{\prime \prime}$. Accordingly, a collection $\mathcal{C} \subseteq 2^{[n]}$ is called strongly (weakly) separated if any two members of $\mathcal{C}$ are strongly (resp. weakly) separated. It is shown in [8] that: (a) any weakly separated collection in $2^{[n]}$ has cardinality at most $\left(\begin{array}{c}n+1 \\ 2\end{array}\right)+1$; and (b) the set $\mathbf{C}_{n}$ of weakly separated collections $\mathcal{C} \subseteq 2^{[n]}$ with $|\mathcal{C}|=\left(\begin{array}{c}n+1 \\ 2\end{array}\right)+1$ includes $\mathcal{B}_{n}$. In fact, the above-mentioned conjecture in [8, Conjecture 1.8] is that this inclusion turns into equality: $\mathbf{C}_{n}=\mathcal{B}_{n}$.

(As is seen from a discussion in [8], an interest in studying weakly separated collections is inspired, in particular, by the problem of characterizing all families of quasicommuting quantum flag minors, which in turn comes from exploration of Lusztig's canonical bases for certain quantum groups. It is proved in [8] that, in an $n \times n$ generic $q$-matrix, the flag minors with column sets $I, J \subseteq[n]$ quasicommute if and only if the sets $I, J$ are weakly separated. See also [7].)

\section{Wirings and tilings}

Wiring and tiling diagrams that we deal with live within a zonogon, which is defined as follows.

In the upper half-plane $\mathbb{R} \times \mathbb{R}_{+}$, take $n$ non-colinear vectors $\xi_{1}, \ldots, \xi_{n}$ so that:

(2.1) (i) $\xi_{1}, \ldots, \xi_{n}$ follow in this order clockwise around $(0,0)$, and

(ii) all integer combinations of these vectors are different.

Then the set

$$
Z=Z_{n}:=\left\{\lambda_{1} \xi_{1}+\ldots+\lambda_{n} \xi_{n}: \lambda_{i} \in \mathbb{R}, 0 \leq \lambda_{i} \leq 1, i=1, \ldots, n\right\}
$$

is a $2 n$-gone. Moreover, $Z$ is a zonogon, as it is the sum of $n$ line-segments $\left\{\lambda \xi_{i}: 1 \leq \lambda \leq 1\right\}, i=$ $1, \ldots, n$. Also it is the image by a linear projection $\pi$ of the solid cube $\operatorname{conv}\left(2^{[n]}\right)$ into the plane $\mathbb{R}^{2}$, defined by $\pi(x):=x_{1} \xi_{1}+\ldots+x_{n} \xi_{n}$. The boundary $b d(Z)$ of $Z$ consists of two parts: the left boundary $l b d(Z)$ formed by the points (vertices) $p_{i}:=\xi_{1}+\ldots+\xi_{i}$ connected by the line-segments $p_{i-1} p_{i}:=$ $p_{i-1}+\left\{\lambda \xi_{i}: 0 \leq \lambda \leq 1\right\}$, and the right boundary $\operatorname{rbd}(Z)$ formed by the points $p_{i}^{\prime}:=\xi_{i+1}+\ldots+\xi_{n}$ connected by the segments $p_{i}^{\prime} p_{i-1}^{\prime}(i=0, \ldots, n)$. So $p_{0}=p_{n}^{\prime}$ is the minimal vertex and $p_{n}=p_{0}^{\prime}$ is the 
maximal vertex of $Z$. We orient each segment $p_{i-1} p_{i}$ from $p_{i-1}$ to $p_{i}$ and orient each segment $p_{i}^{\prime} p_{i-1}^{\prime}$ from $p_{i}^{\prime}$ to $p_{i-1}^{\prime}$. Let $s_{i}$ (resp. $s_{i}^{\prime}$ ) denote the median point in the segment $p_{i-1} p_{i}$ (resp. $p_{i}^{\prime} p_{i-1}^{\prime}$ ).

Although the generalized tiling model will be used more extensively later on, we prefer to start with describing the special wiring model, which looks more transparent.

\subsection{Wiring diagrams}

A special wiring diagram, also called a $W$-diagram or a wiring for brevity, is a collection $W$ of $n$ wires $w_{1}, \ldots, w_{n}$ satisfying three axioms below. A wire $w_{i}$ is a continuous injective map of the segment $[0,1]$ into $Z$ (or the curve in the plane induced by this map) such that $w_{i}(0)=s_{i}, w_{i}(1)=s_{i}^{\prime}$, and $w_{i}(\lambda)$ lies in the interior of $Z$ for $0<\lambda<1$. We say that $w_{i}$ begins at $s_{i}$ and ends at $s_{i}^{\prime}$, and orient $w_{i}$ from $s_{i}$ to $s_{i}^{\prime}$. The diagram $W$ is considered up to a homeomorphism of $Z$ stable on $b d(Z)$, and up to parameterizations of the wires. Axioms (W1)-(W3) specify $W$ as follows.

(W1) No three different wires $w_{i}, w_{j}, w_{k}$ have a common point, i.e., there are no $\lambda, \lambda^{\prime}, \lambda^{\prime \prime}$ such that $w_{i}(\lambda)=w_{j}\left(\lambda^{\prime}\right)=w_{k}\left(\lambda^{\prime \prime}\right)$. Any two different wires $w_{i}, w_{j}$ intersect at a finite number of points, and at each of their common points $v$, the wires cross, not touch (i.e., when passing $v$, the wire $w_{i}$ goes from one connected component of $Z-w_{j}$ to the other).

(W2) for $1 \leq i<j \leq n$, the common points of $w_{i}, w_{j}$ follow in opposed orders along these wires, i.e., if $w_{i}\left(\lambda_{q}\right)=w_{j}\left(\lambda_{q}^{\prime}\right)$ for $q=1, \ldots, r$ and if $\lambda_{1}<\ldots<\lambda_{r}$, then $\lambda_{1}^{\prime}>\ldots>\lambda_{r}^{\prime}$.

Since the order of $s_{i}, s_{j}$ in $\ell b d(Z)$ is different from the order of $s_{i}^{\prime}, s_{j}^{\prime}$ in $\operatorname{rbd}(Z)$, wires $w_{i}, w_{j}$ always intersect; moreover, the number $r=r_{i j}$ of their common points is odd. Assuming that $i<j$, we denote these points as $x_{i j}(1), \ldots, x_{i j}(r)$ following the direction of $w_{i}$. When $r>1$, the region in the plane surrounded by the pieces of $w_{i}, w_{j}$ between $x_{i j}(q)$ and $x_{i j}(q+1)$ (where $q=1, \ldots, r-1$ ) is denoted by $L_{i j}(q)$ and called the $q$-th lens for $w_{i}, w_{j}$. The points $x_{i j}(q)$ and $x_{i j}(q+1)$ are regarded as the lower and upper points of $L_{i j}(q)$, respectively. When $q$ is odd (even), we say that $L_{i j}(q)$ is an odd (resp. even) lens. Note that at each point $x_{i j}(q)$ with $q$ odd the wire with the bigger number, namely, $w_{j}$, crosses the wire with the smaller number $\left(w_{i}\right)$ from left to right w.r.t. the direction of the latter; we call such a point white. In contrast, when $q$ is even, $w_{j}$ crosses $w_{i}$ at $x_{i j}(q)$ from right to left; in this case, we call $x_{i j}(q)$ black, or orientation-reversing, and say that this point is the root of the lenses $L_{i j}(q-1)$ and $L_{i j}(q)$. In the simplest case, when any two distinct wires intersect exactly once, there are no lenses at all and all intersection points for $W$ are white. (The adjectives "white" and "black" for intersection points of wires will match terminology that we use for corresponding elements of tilings.)

The wiring $W$ is associated, in a natural way, with a planar directed graph $G_{W}$ embedded in $Z$. The vertices of $G_{W}$ are the points $p_{i}, p_{i}^{\prime}, s_{i}, s_{i}^{\prime}$ and the intersection points of wires. The edges of $G_{W}$ are the corresponding directed line-segments in $b d(Z)$ and the pieces of wires between neighboring points of intersection with other wires or with the boundary, which are directed according to the direction of wires. We say that an edge contained in a wire $w_{i}$ has color $i$, or is an $i$-edge. Let $\mathcal{F}_{W}$ be the set of (inner) faces of $G_{W}$. Here each face $F$ is considered as the closure of a maximal connected component in $Z-\cup(w \in W)$. We say that a face $F$ is cyclic if its boundary $b d(F)$ is a directed cycle in $G_{W}$.

(W3) There is a bijection $\phi$ between the set $\mathcal{L}(W)$ of lenses in $W$ and the set $\mathcal{F}_{W}^{c y c}$ of cyclic faces in $G_{W}$. Moreover, for each lens $L, \phi(L)$ is the (unique) face lying in $L$ and containing its root. 
We say that $W$ is proper if none of cyclic faces is a whole lens, i.e., for each lens $L \in \mathcal{L}(W)$, there is at least one wire going across $L$. An instance of proper wirings for $n=4$ is illustrated in the picture; here the cyclic faces are marked by circles and the black rhombus indicates the unique black point.

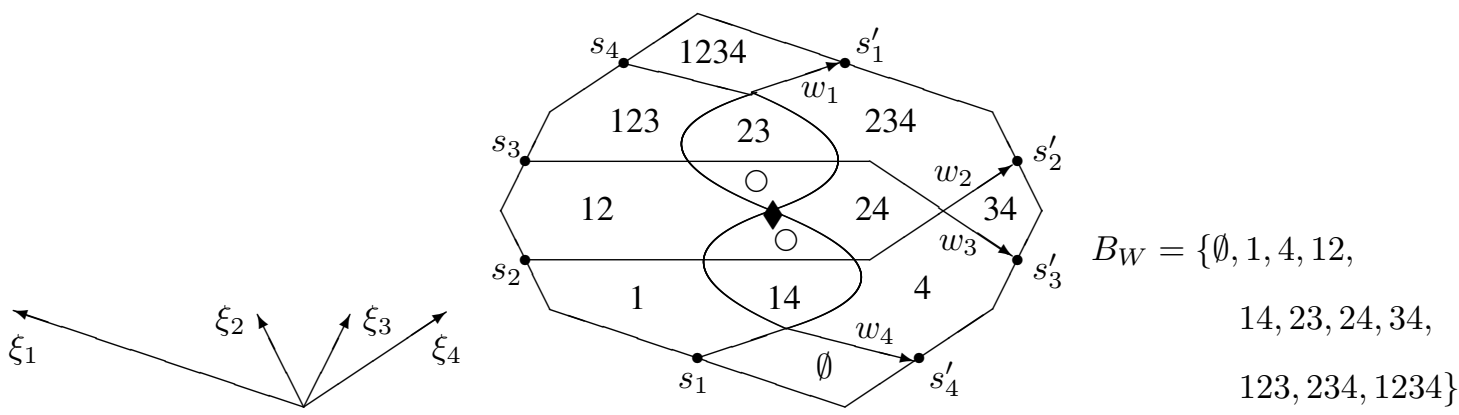

Now we associate to $W$ a set-system $B_{W} \subseteq 2^{[n]}$ as follows. For each face $F$, let $X(F)$ be the set of elements $i \in[n]$ such that $F$ lies on the left from the wire $w_{i}$, i.e., $F$ and the maximal point $p_{n}$ lie in the same of the two connected components of $Z-w_{i}$. We define

$$
B_{W}:=\left\{X \subseteq[n]: X=X(F) \text { for some } F \in \mathcal{F}_{W}-\mathcal{F}_{W}^{c y c}\right\}
$$

referring to it as the effective spectrum, or simply the spectrum of $W$; this is just the object occurring in (ii) of Theorem 1.1. Sometimes it is also useful to consider the full spectrum $\widehat{B}_{W}$ consisting of all sets $X(F), F \in \mathcal{F}_{W}$. (One proves that when $W$ is proper, all sets in $\widehat{B}_{W}$ are different. When $W$ is not proper, there are different faces $F, F^{\prime}$ with $X(F)=X\left(F^{\prime}\right)$. One can turn $W$ into a proper wiring $W^{\prime}$ by getting rid of lenses forming faces (by making a series of Reidemeister moves of type II: $l \rightarrow$ ) (operations). This preserves the effective spectrum: $B_{W^{\prime}}=B_{W}$, whereas the full spectrum may decrease.)

Note that when any two wires intersect at exactly one point (i.e., when no black points exist), $B_{W}$ is a normal basis, and conversely, any normal basis is obtained in this way (see [2]).

\subsection{Generalized tilings}

When it is not confusing, we identify a subset $X \subseteq[n]$ with the corresponding vertex of the $n$-cube and with the point $\sum_{i \in X} \xi_{i}$ in the zonogon $Z$. Due to $\sqrt{2.1}$ (ii), all such points in $Z$ are different.

Assuming that the vectors $\xi_{i}$ have the same Euclidean norm, a rhombus tiling diagram is a subdivision $T$ of $Z$ into rhombi of the form $x+\left\{\lambda \xi_{i}+\lambda^{\prime} \xi_{j}: 0 \leq \lambda, \lambda^{\prime} \leq 1\right\}$ for some $i<j$ and some point $x$ in $Z$, i.e., the rhombi are pairwise non-overlapping (have no common interior points) and their union is $Z$. It follows that for $i, j, x$ as above, $x$ represents a subset in $[n]-\{i, j\}$. We associate to $T$ the directed planar graph $G_{T}$ whose vertices and edges are the vertices and side segments of the rhombi, respectively. An edge connecting $X$ and $X i$ is directed from the former to the latter. It is shown in [2, 4] that the vertex set of $G_{T}$ forms a normal basis and that each normal basis is obtained in this way.

In fact, it makes no difference whether we take vectors $\xi_{1}, \ldots, \xi_{n}$ with equal or arbitrary norms (subject to (2.1); to simplify technical details and visualization, we further assume that these vectors have unit height, i.e., each $\xi_{i}$ is of the form $(x, 1)$. Then we obtain a subdivision $T$ of $Z$ into parallelograms of height 2 , and for convenience refer to $T$ as a tiling and to its elements as tiles. A tile $\tau$ defined by $X, i, j$ (with $i<j$ ) is called an $i j$-tile at $X$ and denoted by $\tau(X ; i, j)$. Its vertices $X, X i, X j, X i j$ are called the bottom, left, right, top vertices of $\tau$ and denoted by $b(\tau), \ell(\tau), r(\tau), t(\tau)$, respectively. 
In a generalized tiling, or a $g$-tiling, the union of tiles is again $Z$ but some tiles may overlap. It is a collection $T$ of tiles partitioned into two subcollections $T^{w}$ and $T^{b}$, of white and black tiles (say), respectively, obeying axioms (T1)-(T4) below. When $T^{b}=\emptyset$, we will obtain a tiling as before, for convenience referring to it as a pure tiling. Let $V_{T}$ and $E_{T}$ denote the sets of vertices and edges, respectively, occurring in tiles of $T$, not counting multiplicities. For a vertex $v \in V_{T}$, the set of edges incident with $v$ is denoted by $E_{T}(v)$, and the set of tiles having a vertex at $v$ is denoted by $F_{T}(v)$.

(T1) All tiles are contained in $Z$. Each boundary edge of $Z$ belongs to exactly one tile. Each edge in $E_{T}$ not contained in $b d(Z)$ belongs to exactly two tiles. All tiles in $T$ are different (in the sense that no two coincide in the plane).

(T2) Any two white tiles having a common edge do not overlap. If a white tile and a black tile share an edge, then these tiles do overlap. No two black tiles share an edge.

(T3) Let $\tau$ be a black tile. None of $b(\tau), t(\tau)$ is a vertex of another black tile. All edges in $E_{T}(b(\tau))$ leave $b(\tau)$ (i.e., are directed from $b(\tau)$ ). All edges in $E_{T}(t(\tau))$ enter $t(\tau)$ (are directed to $t(\tau)$ ).

We distinguish between three sorts of vertices by saying that $v \in V_{T}$ is: (a) a terminal vertex if it is the bottom or top vertex of some black tile; (b) an ordinary vertex if all tiles in $F_{T}(v)$ are white; and (c) a mixed vertex otherwise (i.e. $v$ is the left or right vertex of some black tile). Note that a mixed vertex may belong, as the left or right vertex, to several black tiles.

Each tile $\tau \in T$ is associated, in a natural way, to a square in the solid cube $\operatorname{conv}\left(2^{[n]}\right)$, denoted by $\sigma(\tau)$ : if $\tau=\tau(X ; i, j)$ then $\sigma(\tau)$ is the convex hull of the points $X, X i, X j, X i j$ in the cube. In view of (T1), the interiors of these squares are disjoint, and $\cup(\sigma(\tau): \tau \in T)$ forms a 2-dimensional surface, denoted by $D_{T}$, whose boundary is the preimage by $\pi$ of the boundary of $Z$. The last axiom is:

(T4) $D_{T}$ is a disc (i.e., is homeomorphic to $\left\{x \in \mathbb{R}^{2}: x_{1}^{2}+x_{2}^{2} \leq 1\right\}$ ).

The reversed g-tiling $T^{r e v}$ of a g-tiling $T$ is formed by replacing each tile $\tau(X ; i, j)$ of $T$ by the tile $\tau([n]-X i j ; i, j)$ (or by changing the orientation of all edges in $E_{T}$, in particular, in $b d(Z)$ ). Clearly (T1)-(T4) remain valid for $T^{\text {rev }}$.

The effective spectrum, or simply the spectrum, of a g-tiling $T$ is the collection $B_{T}$ of (the subsets of $[n]$ represented by) non-terminal vertices in $G_{T}$; this is just the object occurring in (iii) of Theorem 1.1 . The full spectrum $\widehat{B}_{T}$ is formed by all vertices in $G_{T}$. An example of g-tilings for $n=4$ is drawn in the picture, where the unique black tile is indicated in bold and the terminal vertices are surrounded by circles (this corresponds to the wiring on the previous picture).

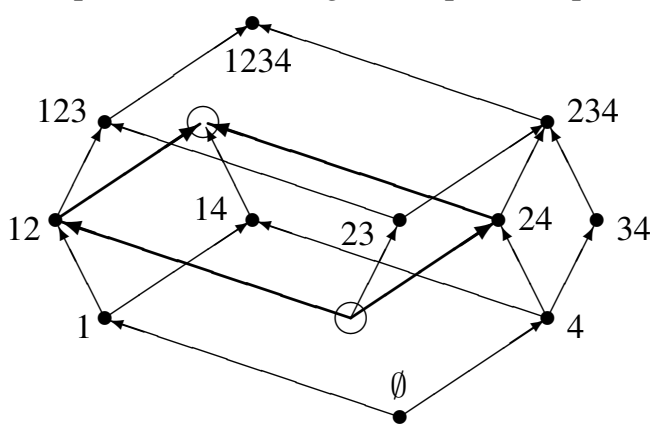

$$
B_{T}=\{\emptyset, 1,4,12,14,23,24,34,
$$


It turns out that for each semi-normal basis $B$, there are precisely one proper wiring $W$ and precisely one g-tiling $T$ such that $B_{W}=B_{T}=B$ (see Theorem 3.3); this is similar to the one-to-one correspondence between the normal bases and pure tilings.

In the rest of this section we point out some (relatively simple) consequences from axioms (T1)-(T4).

1. For a g-tiling $T$, an edge $e$ of the graph $G_{T}=\left(V_{T}, E_{T}\right)$ is called black if there is a black tile containing $e$ (as a side edge); otherwise $e$ is called white. The sets of white and black edges incident with a vertex $v$ are denoted by $E_{T}^{w}(v)$ and $E_{T}^{b}(v)$, respectively. For a vertex $v$ of a tile $\tau$, let $C(\tau, v)$ denote the minimal cone at $v$ containing $\tau$ (i.e., generated by the pair of edges of $\tau$ incident to $v$ ), and let $\alpha(\tau, v)$ denote the angle of this cone taken with sign + if $\tau$ is white, and - if $\tau$ is black. The full rotation angle at $v$ is the sum $\sum\left(\alpha(\tau, v): \tau \in F_{T}(v)\right)$, denoted by $\rho(v)$. The terminal vertices behave as follows.

Corollary 2.1 Let $v$ be a terminal vertex belonging to a black ij-tile $\tau$. Then:

(i) $v$ is not connected by edge with another terminal vertex (whence $\left|E_{T}^{b}(v)\right|=2$ );

(ii) $\left|E_{T}(v)\right| \geq 3$ (whence $E_{T}^{w}(v) \neq \emptyset$ );

(iii) each edge e $\in E_{T}^{w}(v)$ lies in the cone $C(\tau, v)$ (whence e is a q-edge for some $i<q<j$ );

(iv) $\rho(v)=0$;

(v) $v$ does not belong to the boundary of $Z$ (whence any tile containing a boundary edge of $Z$ is white).

2. The rotation angles at non-terminal vertices behave as follows (this is proved by using Euler formula applied to the planar embedding of $G_{T}$ in the disc $D_{T}$ ).

Lemma 2.2 Let $v \in V_{T}$ be a non-terminal vertex.

(i) If $v$ is in $b d(Z)$, then $\rho(v)$ is equal to the angle between the boundary edges incident to $v$.

(ii) If $v$ is inner (i.e., not in $b d(Z)$ ), then $\rho(v)=2 \pi$.

Remark One shows that if property (ii) in Lemma 2.2 is postulated as axiom (T4') and added to axioms (T1)-(T3), then one can eliminate axiom (T4); in other words, (T4') and (T4) are equivalent subject to (T1)-(T3). Note that each of axioms (T1)-(T3),(T4') is "local"; due to Theorem 1.1 this gives rise to a local characterization for the semi-normal TP-bases.

3. An important fact following immediately from 2.1)(ii) is that for any g-tiling $T$, the graph $G_{T}$ is graded for each color $i \in[n]$, which means that for any closed path $P$ in $G_{T}$, the numbers of forward $i$-edges and backward $i$-edges in $P$ are equal.

\section{Ideas of proofs}

As mentioned in the Introduction, the proof of Theorem 1.1 falls into four stages, each consisting in showing one of the implications involved there. Below we outline ideas of our approach.

\subsection{From semi-normal bases to generalized tilings}

The first stage is devoted to showing that any semi-normal TP-basis is representable as the spectrum of some g-tiling, yielding (i) $\rightarrow$ (iii) in Theorem 1.1 .

Consider a g-tiling $T$ on the zonogon $Z=Z_{n}$. By an $M$-configuration in $T$ we mean a quintuple of vertices of the form $X i, X j, X k, X i j, X j k$ with $i<j<k$ (as it resembles the letter "M"), which is denoted as $C M(X ; i, j, k)$. By a $W$-configuration in $T$ we mean a quintuple of vertices $X i, X k, X i j, X i k, X j k$ with $i<j<k$ (as resembling "W"), denoted as $C W(X ; i, j, k)$. A configuration is called feasible if all five vertices are non-terminal, i.e., they belong to $B_{T}$. 
Since any normal basis $B$ (in particular, $B=\mathcal{I}_{n}$ ) is expressed as $B_{T}$ for some pure tiling $T$, it suffices to prove the following assertion saying that the set of g-tilings is closed under transformations analogous to flips for semi-normal bases.

Proposition 3.1 Let a g-tiling T contain five non-terminal vertices $X i, X k, X i j, X j k, Y$, where $i<j<$ $k$ and $Y \in\{X i k, X j\}$. Then there exists a g-tiling $T^{\prime}$ such that $B_{T^{\prime}}$ is obtained from $B_{T}$ by replacing $Y$ by the other member of $\{X i k, X j\}$.

To prove this, one may assume that $Y=X i k$, in which case we have a feasible W-configuration $C W(X ; i, j, k)$ (since any M-configuration in $T$ turns into a W-configurations in the reversed g-tiling $T^{r e v}$ ). We rely on the following two facts (whose proofs are nontrivial).

(P1) Any pair of non-terminal vertices $X^{\prime}, X^{\prime} i^{\prime}$ in $G_{T}$ is connected by edge.

(Note that vertices $X^{\prime}, X^{\prime} i^{\prime}$ need not be connected by edge if some of them is terminal.) So, by (P1), $G_{T}$ contains the edges $(X i, X i j),(X i, X i k),(X k, X i k)$ and $(X k, X j k)$.

(P2) There exist two white tiles $\tau, \tau^{\prime}$ in $T$ such that $\tau$ contains the edges $(X i, X i j)$ and $(X i, X i k)$, and $\tau^{\prime}$ contains the edges $(X k, X i k)$ and $(X k, X j k)$. (These $\tau, \tau^{\prime}$ share the edge $(X i k, X i j k)$.

In addition, one shows (which is not difficult) that the vertex $v:=X i k$ is ordinary.

Based on the these facts, the construction of the desired g-tiling $T^{\prime}$ is as follows. Let $e_{0}, \ldots, e_{q}$ be the sequence of edges entering $v$ in the counterclockwise order. Since $v$ is ordinary, $e_{0}=(X i, X i k)$ and $e_{q}=(X k, X i k)$, and each pair $e_{p-1}, e_{p}(p=1, \ldots, q)$ belongs to a white tile $\tau_{p}$. We consider two possible cases, each case being divided into two subcases.

Case 1: The edges $e:=(X i j, X i j k)$ and $e^{\prime}:=(X j k, X i j k)$ do not belong to the same black tile.

Subcase 1a: $q=1$. We replace in $T$ the tiles $\tau, \tau^{\prime}, \tau_{1}$ by three new white tiles: $\tau(X ; i, j), \tau(X ; j, k)$ and $\tau(X j ; i, k)$ (so the vertex $v$ is replaced by $X j$ ). See the picture.

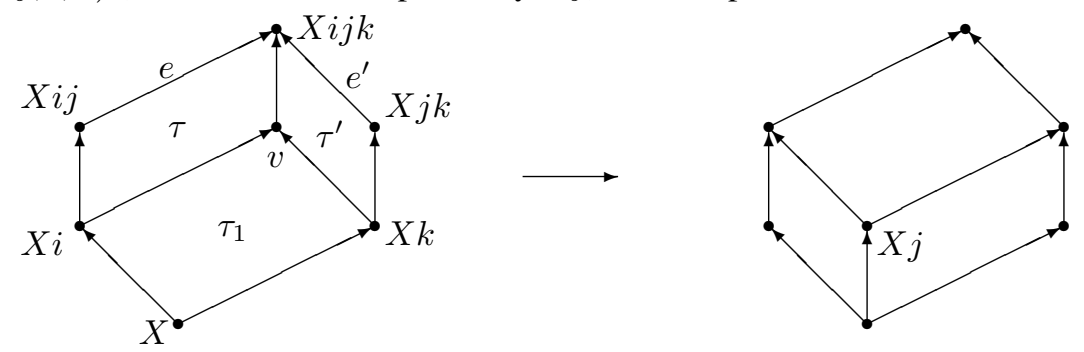

Subcase $1 b: q>1$. We remove the tiles $\tau, \tau^{\prime}$ and add four new tiles: the white tiles $\tau(X ; i, j)$, $\tau(X ; j, k), \tau(X j ; i, k)$ (as before) and the black tile $\tau(X ; i, k)$ (so $v$ becomes terminal). See the picture; here $q=3$ and the added black tile is indicated in bold.

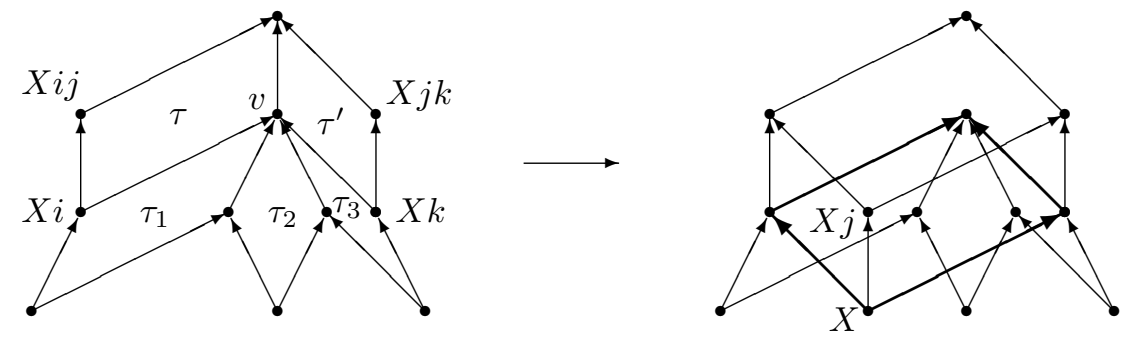


Case 2: Both edges $e$ and $e^{\prime}$ belong to a black tile $\bar{\tau}$ (which is $\tau(X j ; i, k)$ ). We act as in Case 1 with the only differences that $\bar{\tau}$ is removed from $T$ and that the white $i k$-tile at $X j$ (which is a copy of $\bar{\tau}$ ) is not added. Then the vertex $X i j k$ vanishes, $v$ either vanishes or becomes terminal, and $X j$ becomes non-terminal. See the picture. Here (a') and (b') concern Subcase 2a: $q=1$, and Subcase 2b: $q>1$, respectively, and the arc above the vertex $X j$ indicates the bottom cone of $\bar{\tau}$ in which some white edges (not indicated) are located.

(a')



(b’)
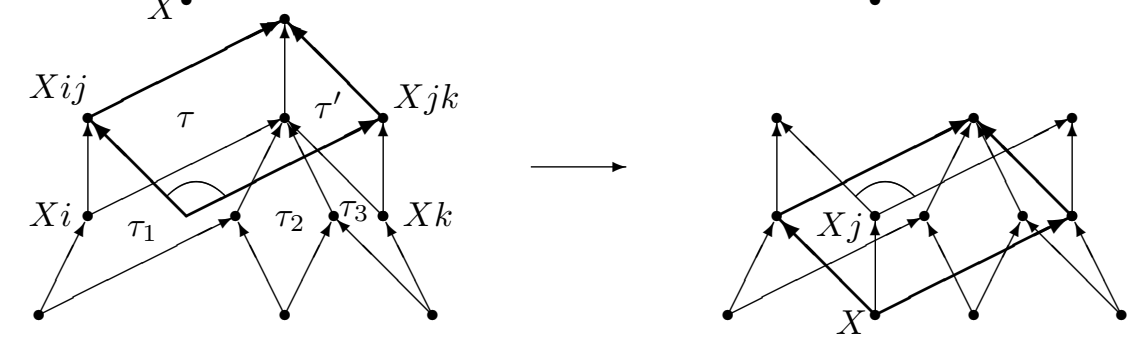

One proves that in all cases the resulting collection $T^{\prime}$ of tiles satisfies axioms (T1)-(T4). Also it is seen from the construction that $B_{T^{\prime}}=\left(B_{T}-\{X i k\}\right) \cup\{X j\}$, as required in Proposition 3.1

\subsection{From generalized tilings to semi-normal bases}

The second stage consists in showing that for any g-tiling $T$, its spectrum $B_{T}$ is a semi-normal TP-basis, yielding (iii) $\rightarrow$ (i) in Theorem 1.1

If $T$ has no black tile, then $\bar{B}_{T}$ is a normal basis, and we are done. So assume $T^{b} \neq \emptyset$. Our aim is to show the existence of a feasible W-configuration $C W(X ; i, j, k)$ for $T$. Then we can transform $T$ into a g-tiling $T^{\prime}$ as in Proposition 3.1, i.e., with $B_{T^{\prime}}=\left(B_{T}-\{X i k\}\right) \cup\{X j\}$. Under such a lowering flip, the sum of sizes of the sets in $B_{\bullet}$ decreases. Then the result will follow by induction on $\sum\left(\left|X^{\prime}\right|: X^{\prime} \in B_{T}\right)$.

By the height $h(v)$ of a vertex $v \in V_{T}$ we mean the size of the corresponding subset of $[n]$. The height $h(\tau)$ of a tile $\tau \in T$ is defined to be the height of its left (or right) vertex.

In fact, we present a sharper version of the desired property.

Proposition 3.2 Let $h \in[n]$. If a g-tiling $T$ has a black tile $\tau$ of height $h$, then there exists a feasible $W$-configuration $C W(X ; i, j, k)$ with $|X|=h-2$. Moreover, such a $C W(X ; i, j, k)$ can be chosen so that Xijk is the top vertex of some black tile (of height $h$ ).

To prove this, starting from $\tau_{0}:=\tau$, choose a vertex $u_{0}$ adjacent to $v_{0}:=t\left(\tau_{0}\right)$ and different from $\ell\left(\tau_{0}\right), r\left(\tau_{0}\right)$ (it exists by (ii) in Corollary 2.1). Then there are white tiles $\tau^{\prime}, \tau^{\prime \prime}$ such that $t\left(\tau^{\prime}\right)=t\left(\tau^{\prime \prime}\right)=$ $v_{0}$ and $r\left(\tau^{\prime}\right)=\ell\left(\tau^{\prime \prime}\right)=u_{0}$. One easily shows that if $u_{0}$ is ordinary, then both vertices $b\left(\tau^{\prime}\right), b\left(\tau^{\prime \prime}\right)$ are non-terminal, implying that these vertices together with $u_{0}, \ell\left(\tau^{\prime}\right), r\left(\tau^{\prime \prime}\right)$ form a feasible W-configuration (as required in the proposition). And if $u_{0}$ is mixed, then there is a black tile $\tau_{1}$ (of the same height $h$ ) having $u_{0}$ as the left or right vertex. We treat $\tau_{1}$ in a similar way as $\tau_{0}$, by choosing a vertex $u_{1}$ adjacent 
to $v_{1}:=t\left(\tau_{1}\right)$ and different from $\ell\left(\tau_{1}\right), r\left(\tau_{1}\right)$. If $u_{1}$ is mixed again, we continue the process. Sooner or later, we obtain a black tile $\tau_{k}$ and a chosen vertex $u_{k}$ such that either $u_{k}$ is ordinary, in which case we are done, or $\tau_{k}$ coincides with $\tau_{0}$. In the latter case, one shows (a key) that there exists $i \in[n]$ such that in the cycle passing $v_{0}, u_{0}, v_{1}, u_{1}, v_{2}, \ldots, u_{k-1}, v_{k}=v_{0}$, the numbers of forward and backward edges with color $i$ are not equal, contrary to the fact that $G_{T}$ is graded.

\subsection{From generalized tilings to proper wirings}

The third stage consists in showing that for any g-tiling $T$ on the zonogon $Z=Z_{n}$, there exists a proper wiring $W$ on $Z$ such that $B_{W}=B_{T}$, yielding (iii) $\rightarrow$ (ii) in Theorem 1.1 .

To prove this, we use the notion of an $i$-strip (or a dual $i$-path) for $T$, where $i \in[n]$. This is a maximal sequence $Q=\left(e_{0}, \tau_{1}, e_{1}, \ldots, \tau_{r}, e_{r}\right)$ of edges and tiles such that: (a) $\tau_{1}, \ldots, \tau_{r}$ are different tiles, each being an $i j$-or $j i$-tile for some $j$, and (b) for $p=1, \ldots, r, e_{p-1}$ and $e_{p}$ are the opposite $i$-edges of $\tau_{p}$ (recall that when speaking of an $i^{\prime} j^{\prime}$-tile, one assumes $i^{\prime}<j^{\prime}$.) Clearly $Q$ is determined uniquely, up to reversing it and shifting cyclically (when $e_{0}=e_{r}$ ), by any of its edges or tiles. Using the fact that $G_{T}$ is graded, one shows that $Q$ cannot be cyclic, i.e., the edges $e_{0}$ and $e_{r}$ are different. Then one of $e_{0}, e_{r}$ lies on the left boundary, and the other on the right boundary of $Z$; we may assume that $e_{0} \in \ell b d(Z)$.

For convenience we identify the tiles in $T$ with the corresponding squares in the disc $D_{T}$ (whose interiors are pairwise disjoint). To construct the desired wiring $W$, each $i$-strip $Q_{i}=\left(e_{0}, \tau_{1}, e_{1}, \ldots, \tau_{r}, e_{r}\right)$ for $T$ is regarded as a sequence of straight-line segments and squares on $D_{T}$, and we draw the "median" piece-wise linear curve $\zeta_{i}$ within $Q_{i}$. More precisely, for $q=1, \ldots, r$, draw the line-segment on $\tau_{q}$ connecting the median points of the edges $e_{r-1}$ and $e_{r}$. This segment meets the central point of $\tau_{q}$, denoted by $c\left(\tau_{q}\right)$. The concatenation of these segments is just $\zeta_{i}$; we direct it according to the direction of $Q_{i}$.

Now fix a homeomorphic map $\gamma: D_{T} \rightarrow Z$ that brings the boundary of $D_{T}$ to $b d(Z)$ in a natural way. This turns the above curves into the wires $w_{i}:=\gamma\left(\zeta_{i}\right)$ on $Z$, where $w_{i}$ begins at the median point $s_{i}$ of $p_{i-1} p_{i}$ on $\ell b d(Z)$ and ends at the medial point $s_{i}^{\prime}$ of $p_{i}^{\prime} p_{i-1}^{\prime}$ on $\left.\operatorname{rbd}(Z)\right)$. We assert that the wiring $W=\left(w_{1}, \ldots, w_{n}\right)$ is as required.

Clearly $W$ satisfies axiom (W1). To verify the other axioms, we first should explain how the planar graphs $G_{T}$ and $H:=\gamma^{-1}\left(G_{W}\right)$ on $D_{T}$ are related to each other. The vertices of $H$ are the central points $c(\tau)$ of squares $\tau$ and the points $s_{i}, s_{i}^{\prime}$ (identifying the boundaries of $D_{T}$ and $Z$ by $\gamma$ ). Each vertex $v$ of $G_{T}$ corresponds to the face of $H$ where $v$ is located, denoted by $v^{*}$. The edges of color $i$ in $H$ correspond to the $i$-edges of $G_{T}$. More precisely, if an $i$-edge $e \in E_{T}$ belongs to squares $\tau, \tau^{\prime}$ and if $\tau, e, \tau^{\prime}$ occur in this order in the $i$-strip, then the $i$-edge $e^{*}$ of $H$ corresponding to $e$ is the piece of $\zeta_{i}$ between $c(\tau)$ and $c\left(\tau^{\prime}\right)$, and this $e^{*}$ is directed from $c(\tau)$ to $c\left(\tau^{\prime}\right)$. Observe that $e$ crosses $e^{*}$ from right to left on the disc. The first (last) piece of $\zeta_{i}$ corresponds to the boundary $i$-edge $p_{i-1} p_{i}$ (resp. $p_{i}^{\prime} p_{i-1}^{\prime}$ ) of $G_{T}$.

Consider an $i j$-tile $\tau \in T$, and let $e, e^{\prime}$ be its $i$-edges, and $u, u^{\prime}$ its $j$-edges, where $e, u$ leave $b(\tau)$ and $e^{\prime}, u^{\prime}$ enter $t(\tau)$. One can see that: (a) if $\tau$ is white, then $e$ occurs in $Q_{i}$ before $e^{\prime}$, while $u$ occurs in $Q_{j}$ after $u^{\prime}$, and (b) if $\tau$ is black, then $e$ occurs in $Q_{i}$ after $e^{\prime}$, while $u$ occurs in $Q_{j}$ before $u^{\prime}$. In the disc $D_{T}$, both $e, e^{\prime}$ cross the wire $\zeta_{i}$ from right to left (w.r.t. the direction of $\zeta_{i}$ ), and similarly both $u, u^{\prime}$ cross $\zeta_{j}$ from right to left. Axioms (T1),(T2) for $T$ imply that when $\tau$ is white, the orientations of $\tau$ in $Z$ and in $D_{T}$ are the same, whereas when $\tau$ is black, the clockwise orientation of $\tau$ in $Z$ turns in the counterclockwise orientation of $\tau$ in $D_{T}$. It follows that: in case (a), $\zeta_{j}$ crosses $\zeta_{i}$ at $c(\tau)$ from left to right, and in case (b), $\zeta_{j}$ crosses $\zeta_{i}$ at $c(\tau)$ from right to left; see the picture. So the white (black) tiles of $T$ generate the white (resp. black) vertices of $G_{W}$. 
(a)

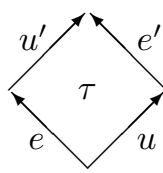

(in $Z$ )



(in $D_{T}$ ) (b)

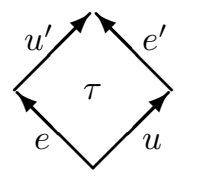

(in $Z$ )

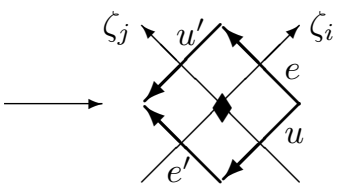

(in $D_{T}$ )

For a vertex $v$ of $G_{T}$ and an edge $e \in E_{T}(v)$, the edge $e^{*}$ belongs to the boundary of the face $v^{*}$ of $H$. Since $e$ crosses $e^{*}$ from right to left, $e^{*}$ is directed clockwise around $v^{*}$ if $e$ leaves $v$, and counterclockwise if $e$ enters $v$. Then axiom (T3) for $T$ implies that the terminal vertices of $G_{T}$ and only them generate cyclic faces of $G_{W}$, yielding validity of (W3).

Next, for each $i \in[n]$, removing from $D_{T}$ the interior of the $i$-strip $Q_{i}$ results in two closed regions $\Omega_{1}, \Omega_{2}$ containing the vertices $\emptyset$ and [n], respectively. Also all edges in $Q_{i}$ go from $\Omega_{1}$ to $\Omega_{2}$, whence each vertex $v$ of $G_{T}$ occurring in $\Omega_{1}\left(\Omega_{2}\right)$ is a subset of $[n]$ not containing (resp. containing) the element $i$. So $i \notin X\left(v^{*}\right)$ if and only if $v \in \Omega_{1}$. This implies the desired equality for spectra: $B_{W}=B_{T}$.

A verification of (W2) for $W$ is less trivial; this is done by using the fact that $G_{T}$ is graded (we omit the details). Finally, since $\left|E_{T}(v)\right| \geq 3$ for each terminal vertex $v$ in $G_{T}$, each cyclic face in $G_{W}$ is surrounded by at least three edges, and therefore, this face cannot be a lens. So the wiring $W$ is proper.

\subsection{From proper wirings to generalized tilings}

The final, fourth, stage is devoted to showing that for any proper wiring $W$ on the zonogon $Z=Z_{n}$, there exists a g-tiling $T$ on $Z$ such that $B_{T}=B_{W}$, yielding (ii) $\rightarrow$ (iii) in Theorem 1.1. The construction of $T$ is converse, in a sense, to that described in the previous subsection; it combines planar duality techniques and geometric arrangements.

We associate to each inner face $F$ of the graph $G_{W}$ the point (viz. the subset) $X(F)$ in the zonogon, also denoted as $F^{*}$. These points are just the vertices of tiles in $T$. The edges of $G_{T}$ are defined as follows. Let faces $F, F^{\prime} \in \mathcal{F}_{W}$ have a common edge $e$ formed by a piece of a wire $w_{i}$, and let $F$ lie on the right from $w_{i}$ according to the direction of this wire. Then the vertices $F^{*}, F^{\prime *}$ are connected by edge $e^{*}$ going from $F^{*}$ to $F^{\prime *}$. Note that in view of the evident relation $X\left(F^{\prime}\right)=X(F) \cup\{i\}$, the direction of $e^{*}$ matches the edge direction for g-tilings.

The tiles in $T$ correspond to the intersection points of wires in $W$. More precisely, let $v$ be a common point of wires $w_{i}, w_{j}$ with $i<j$. Then the vertex $v$ of $G_{W}$ has four incident edges $e_{i}, \bar{e}_{i}, e_{j}, \bar{e}_{j}$ such that: $e_{i}, \bar{e}_{i} \subset w_{i} ; e_{j}, \bar{e}_{j} \subset w_{j} ; e_{i}, e_{j}$ enter $v$; and $\bar{e}_{i}, \bar{e}_{j}$ leave $v$. One can see that for the four faces $F$ containing $v$, the subsets $X(F)$ are of the form $X, X i, X j, X i j$ for some $X \subset[n]$. The tile surrounded by the edges $e_{i}^{*}, \bar{e}_{i}^{*}, e_{j}^{*}, \bar{e}_{j}^{*}$ connecting these subsets (regarded as points) is just the $i j$-tile in $T$ corresponding to $v$, denoted as $v^{*}$. Observe that the edges $e_{i}, e_{j}, \bar{e}_{i}, \bar{e}_{j}$ follow in this order clockwise around $v$ if $v$ is white, and counterclockwise if $v$ is black. According to this, the tile $v^{*}$ is assigned to be white in $T$ if $v$ is white, and black otherwise. Both cases are illustrated in the picture:
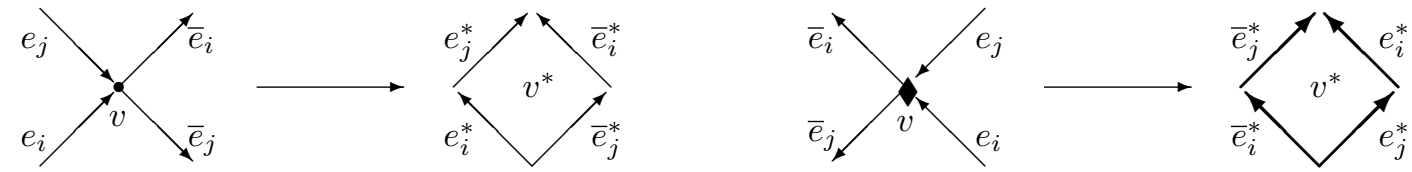

A proof that $T$ is indeed a correct g-tiling on $Z$ and that $B_{T}=B_{W}$ consists of several verifications of which some are not straightforward; we omit the details. 
Properties of g-tilings and proper wirings established during the whole proof of Theorem 1.1 enable us to obtain the following rigidity result.

Theorem 3.3 For each semi-normal basis $B$, there are a unique g-tiling $T$ and a unique proper wiring $W$ such that $B=B_{T}=B_{W}$.

Also one can offer an efficient (polynomial-time) algorithm that, given a collection $B \subset 2^{[n]}$, decides whether $B$ is representable as the spectrum of a g-tiling, and if so, explicitly constructs this g-tiling.

\section{References}

[1] A. Berenstein, S. Fomin, and A. Zelevinsky, Parametrizations of canonical bases and totally positive matrices, Adv. Math. 122 (1996), 49-149.

[2] V. Danilov, A. Karzanov and G. Koshevoy, On bases of tropical Plücker functions, ArXiv:0712.3996[math.CO], 2007.

[3] V. Danilov, A. Karzanov and G. Koshevoy, Plücker environments, wiring and tiling diagrams, and weakly separated set-systems, ArXiv:0902.3362v3[math.CO], 2009.

[4] A. Henriques and D.E. Speyer, The multidimensional cube recurrence, ArXiv:0708.2478v1[math.CO], 2007.

[5] J. Kamnitzer, The crystal structure on the set of Mirkovic-Vilonen polytopes, Adv. Math. 215 (1) (2007), 66-93.

[6] R. Kenyon and J.-M. Schlenker, Rhombic embeddings of planar graphs with faces of degree 4, ArXiv:math-ph/0305057, 2003.

[7] A. Lauve, Quasideterminants and q-commuting minors, ArXiv:0602.448 [math.QA], 2006.

[8] B. Leclerc and A. Zelevinsky: Quasicommuting families of quantum Plücker coordinates, Amer. Math. Soc. Trans., Ser. 2181 (1998), 85-108.

[9] A. Postnikov, Total positivity, Grassmannians, and networks, ArXiv:math.CO/0609764, 2006.

[10] J. Scott, Grassmannians and cluster algebras, Proc. London Math. Soc. (3) 92 (2) (2006), 345-380.

[11] D. Speyer, Perfect matchings and the octahedron recurrence, J. Algebraic Combin. 25 (2007), 309348.

[12] D. Speyer, L. Williams, The tropical totally positive Grassmanian, J. Algebraic Combin. 22 (2) (2005), 189-210. 